\title{
About Total Stability of a Class of Nonlinear Dynamic Systems Eventually Subject to Discrete Internal Delays
}

\author{
Manuel De la Sen (iD) \\ Institute of Research and Development of Processes IIDP, University of the Basque Country, Campus of Leioa, P.O. Box 48940, \\ Leioa, Bizkaia, Spain
}

Correspondence should be addressed to Manuel De la Sen; manuel.delasen@ehu.eus

Received 28 January 2021; Accepted 23 February 2021; Published 4 March 2021

Academic Editor: Xiaodi Li

Copyright (C) 2021 Manuel De la Sen. This is an open access article distributed under the Creative Commons Attribution License, which permits unrestricted use, distribution, and reproduction in any medium, provided the original work is properly cited.

This paper studies and investigates total stability results of a class of dynamic systems within a prescribed closed ball of the state space around the origin. The class of systems under study includes unstructured nonlinearities subject to multiple higher-order Lipschitz-type conditions which influence the dynamics and which can be eventually interpreted as unstructured perturbations. The results are also extended to the case of presence of multiple internal (i.e., in the state) point discrete delays. Some stability extensions are also discussed for the case when the systems are subject to forcing efforts by using links between the controllability and stabilizability concepts from control theory and the existence of stabilizing linear controls. The results are based on the ad hoc use of Gronwall's inequality.

\section{Introduction}

The study of the stability properties is of major interest in dynamic systems since it allows to investigate the existence and nature of the equilibrium points, their respective domains of attraction, and the eventual existence of oscillations dependent on the initial conditions or asymptotic oscillations independent on the initial conditions (limit cycles). Typical techniques of investigation of stability are those based on local and global Lyapunov stability methods and Bendixson-type theorems for investigation of the oscillatory and asymptotic oscillatory behaviors (see, for instance, $[1-16]$ and references therein). There are also frequency-type methods of stability analysis, such as, Routh-Hurwitz criterion, Jury criterion, amplitude and phase Bode plots, Nyquist criterion, root locus, Tsypkin locus, and describing function analysis, which are very popular in the time-invariant case and also in systems including some specific types of separate nonlinearities in actuators or sensors such as, for instance, ban-bang (i.e., relay) or saturating devices (see, for instance, [4-6]). Also, the wellknown Gronwall inequality in its various versions can be adapted to stability studies in the time-domain (see, for instance, $[1,2])$.
On the other hand, it can be noticed that delays are present in a natural way in the real life and in many real processes such as, for instance, in some biological equations as, for instance, in epidemic models, in some generalizations of the Beverton-Holt equation, in prey-predator problems, or in the sunflower equation and in population growing and diffusion problems. They are also present in many engineering diffusion problems, information package transmission, queuing storages, teleoperation systems, chemical processes, and robotic systems. The study of stability of timedelay systems has received much attention in the last decades. For instance, a nonautonomous functional differential equation of the third order is considered in [7] with multiple deviating arguments. Asymptotic stability results and uniform boundedness of the solution results are obtained based on the ad hoc use of the Lyapunov-Krasovskii functional approach in the above paper. Other stability results for third-order differential equations are proved in $[9,10]$. Also, some sufficiency-type conditions of global stability and asymptotic stability results for time-varying systems with state-dependent parameterizations are obtained in [8]. On the other hand, other studies of stability and robustness of dynamic systems have been performed in [11-14] and related studies for the 
presence of oscillatory behaviors have been performed in $[15,16]$ and references therein. The study of stabilization of time-delay dynamic systems and/or integrodifferential equations has been also addressed to more general classes of equations. For instance, the exponential decay of a first-order linear Volterra equation is investigated in [17] while the exponential stability of controlled nonlinear systems subject to time-varying delays has been discussed in [18]. It can be noticed that Volterra-type equations can be interpreted as being subject to a distributed delay on their previous evolution history. On the other hand, the solvability and optimal controls of a noninstantaneous impulsive neutral stochastic integer-differential equation forced by Brownian motion are addressed in [19]. It can be noticed that neutral differential equations are a class of retarded equations where the highestorder time-derivative is affected by delays contrarily to the case of nonneutral ones.

This paper gives some results on total stability of a class of nonlinear time-varying systems subject to combined multiple higher-order-type Lipschitz conditions. The stability results are of global type within some prescribed closed ball around the origin and are subject to conditions of exponential stability of the nonlinearity-free system counterpart and to maximum bounds of the various Lipschitz constants. Some applications are given for the control case. The above results are included in Section 2. On the other hand, Section 3 extends the results of Section 2 for the case of presence of multiple punctual constant delays which appear jointly in the linear dynamics of the system and in the nonlinear one which is also subject to combined multiple higher-order-type Lipschitz conditions.

\section{Total Stability of Nonlinear Differential Systems with Forcing Terms Subject to Multiple Combined Higher-Order Lipschitz Conditions}

The following result is two-fold since it relies on the uniqueness of the solution exhibiting, furthermore, global stability in a bounded region around the origin for a class of nonlinear time-varying differential systems under a set of Lipschitz-extended power-type perturbations of the state. A combined analysis supported by the Banach Contraction Principle and Bellman-Gronwall Lemma [1-3] is used to get the next main result.

Theorem 1. Consider the following differential system:

$$
\dot{x}(t)=F(t, x),
$$

subject to initial conditions $x\left(t_{0}\right)=x_{0}$ where $F:\left[t_{0}, \infty\right) \times$ $\mathbf{R}^{n} \longrightarrow \mathbf{R}^{n}$ is given by

$$
F(t, x)=A(t) x(t)+\sum_{i=1}^{p} f_{i}(t, x)+\sum_{i=1}^{q} g_{i}(t, x),
$$

where $A:\left[t_{0}, \infty\right) \longrightarrow \mathbf{R}^{n \times n}$ has bounded piecewise continuous entries and $f_{i}, g_{j}:\left[t_{0}, \infty\right) \times \mathbf{R}^{n} \longrightarrow \mathbf{R}^{n} ; i=1,2, \ldots, p$; $j=1,2, \ldots, q$ are locally integrable functions of $t$ on $\left[t_{0}, \infty\right]$ in the closed ball $B_{r}=\left\{x \in \mathbf{R}^{n}:\|x\| \leq r\right\}$, where $\|x\|$ denotes some vector norm, and suppose that the following two assumptions hold:

(A1) $f_{i}(t, 0)=0$ and $\left\|f_{i}\left(t, x_{1}\right)-f_{i}\left(t, x_{2}\right)\right\| \leq \beta_{i} \| x_{1}$ $-x_{2} \|^{i} ; i=1,2, \ldots, p$ for all $x_{1}, x_{2} \in B_{r}$

(A2) $\left\|g_{i}\left(t, x_{1}\right)\right\| \leq \gamma_{i} r^{i+1}$ and $\left\|g_{i}\left(t, x_{1}\right)-g_{i}\left(t, x_{2}\right)\right\| \leq$ $\gamma_{i}\left\|x_{1}-x_{2}\right\|^{i} ; i=1,2, \ldots, q$ for all $x_{1}, x_{2} \in B_{r}$

(A3) The auxiliary unforced differential system

$$
\begin{aligned}
\dot{z}(t) & =A(t) z(t), \\
z\left(t_{0}\right) & =z_{0},
\end{aligned}
$$

is exponentially stable on $\left[t_{0}, \infty\right)$ of stability abscissa $-\alpha<0$; that is, there exist real constants $\alpha>0$ and $K \geq 1$ such that the fundamental matrix $\Psi:\left[t_{0}, \infty\right] \longrightarrow \mathbf{R}^{n}$, which is the mild solution of $\dot{\psi}\left(t, t_{0}\right)=A(t) \psi\left(t, t_{0}\right)$ for $t \geq t_{0}$ with $\psi\left(t_{0}, t_{0}\right)=I_{n} \quad\left(I_{n}\right.$ being the $n$-th identity matrix) satisfies $\left\|\Psi\left(t_{1}, t_{2}\right)\right\| \leq K e^{-\alpha\left(t_{2}-t_{1}\right)}$ for any $t_{1}$ and $t_{2}$ such that $t_{2} \geq t_{1} \geq t_{0}$.

(A4) The constants $\beta_{i}$ and $\gamma_{j}, i=1,2, \ldots, p$ and $j=1,2, \ldots, q$, satisfy

$$
\sum_{i=1}^{p} \beta_{i} r^{i-1}+\sum_{i=1}^{q} \gamma_{i} r^{i-1} \leq \frac{\alpha}{K}
$$

for some given $r>0$.

Then, the following properties hold:

(i) $\sup _{t_{0} \leq t \leq \infty}\|x(t)\| \leq r$ if $\left\|x_{0}\right\| \leq(r / K)$ so that differential systems (1) and (2) are globally Lyapunov stable in the closed ball of radius $r$ centred at the origin.

(ii) $\limsup _{t \longrightarrow \infty}\|x(t)\| \leq \frac{K r}{\alpha}\left(\sum_{i=1}^{p} \beta_{i} r^{i-1}+\sum_{i=1}^{q} \gamma_{i} r^{i-1}\right) \leq r$,

for any given finite $\left\|x_{0}\right\|$.

(iii) Assume that A4 is restricted to a strict inequality, that is,

$$
\text { (A5) } \sum_{i=1}^{p} \beta_{i} r^{i-1}+\sum_{i=1}^{q} \gamma_{i} r^{i-1}<\frac{\alpha}{K},
$$

for some given $r>0$. Then, there is a unique globally Lyapunov stable solution of (1) and (2) in the closed ball of radius $r$ centred at the origin for $t \geq t_{0}$ under Assumptions A1, A2, A3, and A5 satisfying

$$
\begin{gathered}
\|x(t)\| \leq K e^{-\left(\alpha-K \sum_{i=1}^{q} \beta_{i} i^{i-1}\right)\left(t-t_{0}\right)}\left\|x_{0}\right\|+\frac{K \sum_{i=1}^{q} \gamma_{i} r^{i}}{\alpha-K \sum_{i=1}^{q} \beta_{i} r^{i-1}} \\
\left(1-e^{-\left(\alpha-K \sum_{i=1}^{q} \beta_{i} r^{i-1}\right)\left(t-t_{0}\right)}\right) \leq r, \quad \forall t \geq t_{0} .
\end{gathered}
$$




$$
x(t)=\Psi\left(t, t_{0}\right) x_{0}+\sum_{i=1}^{p} \int_{t_{0}}^{t} \Psi(t, \tau) f_{i}(\tau, x(\tau)) \mathrm{d} \tau+\sum_{i=1}^{q} \int_{t_{0}}^{t} \Psi(t, \tau) g_{i}(\tau, x(\tau)) \mathrm{d} \tau
$$

and, by using Assumptions A1 to A3, one gets

$$
\begin{aligned}
& \|x(t)\| \leq K e^{-\alpha\left(t-t_{0}\right)}\left\|x_{0}\right\|+K\left(\sum_{i=1}^{p} \beta_{i} r^{i}+\sum_{i=1}^{q} \gamma_{i} r^{i}\right)\left(\int_{t_{0}}^{t} e^{-\alpha(t-\tau)} \mathrm{d} \tau\right), \\
& =K e^{-\alpha\left(t-t_{0}\right)}\left\|x_{0}\right\|+K\left(\sum_{i=1}^{p} \beta_{i} r^{i-1}+\sum_{i=1}^{q} \gamma_{i} r^{i-1}\right) \frac{r}{\alpha}\left(1-e^{-\alpha\left(t-t_{0}\right)}\right), \\
& =K e^{-\alpha\left(t-t_{0}\right)}\left(\left\|x_{0}\right\|-\frac{r}{\alpha}\left(\sum_{i=1}^{p} \beta_{i} r^{i-1}+\sum_{i=1}^{q} \gamma_{i} r^{i-1}\right)\right)+\frac{K r}{\alpha}\left(\sum_{i=1}^{p} \beta_{i} r^{i-1}+\sum_{i=1}^{q} \gamma_{i} r^{i-1}\right) \leq r, \quad t \geq t_{0},
\end{aligned}
$$

provided that A4 holds subject to

$$
K\left\|x_{0}\right\| \leq\left(\frac{K r}{\alpha}-\frac{K r}{\alpha}\right)\left(\sum_{i=1}^{p} \beta_{i} r^{i-1}+\sum_{i=1}^{q} \gamma_{i} r^{i-1}\right)+r=r,
$$

which is got from (9) for $t=t_{0}$. Then, Property (i) is proved. On the other hand, since $\alpha>0$, one gets from A4 and (9) that
(5) holds and Property (ii) is proved. Now, let $C^{n}\left[t_{0}, \infty\right)$ be the Banach space of continuous, bounded $n$-vector real functions $y:\left[t_{0}, \infty\right) \longrightarrow \mathbf{R}^{n}$ equipped with the norm \|\|$y\left\|\left.\right|_{\left[t_{0}, \infty\right)}=\sup _{t_{0} \leq t<\infty}\right\| x(t) \|$. Let $T$ an operator on $C^{n}\left[t_{0}, \infty\right)$ represent solution (1) which is defined pointwise via

$$
(T x)(t)=\Psi\left(t, t_{0}\right) x_{0}+\sum_{i=1}^{p} \int_{t_{0}}^{t} \Psi(t, \tau) f_{i}(\tau, x(\tau)) \mathrm{d} \tau+\sum_{i=1}^{q} \int_{t_{0}}^{t} \Psi(t, \tau) g_{i}(\tau, x(\tau)) \mathrm{d} \tau
$$

for $t \geq t_{0}$. Then, if $\left\|x_{0}\right\| \leq(r / K)$, one has from (9) that

$$
\|(T x)(t)\| \leq K e^{-\alpha\left(t-t_{0}\right)}\left\|x_{0}\right\|+\frac{K r}{\alpha}\left(\sum_{i=1}^{p} \beta_{i} r^{i-1}+\sum_{i=1}^{q} \gamma_{i} r^{i-1}\right) \leq r,
$$

and one has then from (8) for any solutions $x_{1}, x_{2} \in B_{r}$ on $\left[t_{0}, \infty\right)$ under the same initial condition $x_{0}$ that

$$
\begin{aligned}
x_{1}(t)-x_{2}(t)= & \sum_{i=1}^{p} \int_{t_{0}}^{t} \Psi(t, \tau)\left(f_{i}\left(\tau, x_{1}(\tau)\right)-f_{i}\left(\tau, x_{2}(\tau)\right)\right) \mathrm{d} \tau \\
& +\sum_{i=1}^{q} \int_{t_{0}}^{t} \Psi(t, \tau)\left(g_{i}\left(\tau, x_{1}(\tau)\right)-g_{i}\left(\tau, x_{2}(\tau)\right)\right) \mathrm{d} \tau,
\end{aligned}
$$

which leads to

$$
\|\|\left(T x_{1}\right)(t)-\left(T x_{2}\right)(t)\left\|\left.\right|_{\left[t_{0}, \infty\right)} \leq \frac{K r}{\alpha}\left(\sum_{i=1}^{p} \beta_{i} r^{i-1}+\sum_{i=1}^{q} \gamma_{i} r^{i-1}\right)\left|\left\|x_{1}(t)-x_{2}(t)\right\|\right|_{\left[t_{0}, \infty\right)},\right.
$$

so that $T: C^{n}\left[t_{0}, \infty\right) \longrightarrow C^{n}\left[t_{0}, \infty\right)$ is a contraction on the closed subset $\mathbf{B}_{r}=\left\{y \in C^{n}\left[t_{0}, \infty\right):\|y\| \leq r\right\}$ of $C^{n}\left[t_{0}, \infty\right)$ if A5 holds. This implies from the contraction mapping theorem that solution (8) is unique. From (11) and Assumptions $\mathrm{A}-\mathrm{A} 3$, one gets

$$
\|x(t)\| \leq K e^{-\alpha\left(t-t_{0}\right)}\left\|x_{0}\right\|+\left(\sum_{i=1}^{p} \beta_{i} r^{i-1}\right)\left(\int_{t_{0}}^{t} e^{-\alpha(t-\tau)}\|x(\tau)\| \mathrm{d} \tau\right)+\left(\sum_{i=1}^{q} \gamma_{i} r^{i}\right)\left(\int_{t_{0}}^{t} e^{-\alpha(t-\tau)} \mathrm{d} \tau\right), \quad t \geq t_{0} .
$$


Also, one has from (15) that $e^{\alpha t}\|x(t)\| \leq \xi(t), t \geq t_{0}$ from for $t \geq t_{0}$, which is Bellman-Gronwall Lemma, where $\xi:\left[t_{0}, \infty\right) \longrightarrow \mathbf{R}$ is the solution of

$$
\begin{aligned}
& \dot{\xi}(t)=K\left[\left(\sum_{i=1}^{p} \beta_{i} r^{i-1}\right) \xi(t)+\left(\sum_{i=1}^{q} \gamma_{i} r^{i}\right) e^{\alpha t}\right], \\
& \xi\left(t_{0}\right)=K e^{\alpha t_{0}}\left\|x_{0}\right\|,
\end{aligned}
$$

$$
\xi(t)=e^{K\left(\sum_{i=1}^{q} \beta_{i} r^{i-1}\right)\left(t-t_{0}\right)} \xi\left(t_{0}\right)+\frac{K \sum_{i=1}^{q} \gamma_{i} r^{i}}{\alpha-K \sum_{i=1}^{q} \beta_{i} r^{i-1}}\left(1-e^{-\left(\alpha-K \sum_{i=1}^{q} \beta_{i} r^{i-1}\right)\left(t-t_{0}\right)}\right) e^{\alpha t}, \quad t \geq t_{0},
$$

which implies (7) and proves Proposition 3.

Some direct corollaries are as follows.

$$
F(t, x)=B(t) x(t)+\sum_{i=1}^{p} f_{i}(t, x)+\sum_{i=1}^{q} g_{i}(t, x)
$$

Corollary 1. Consider differential system (1) with

with $B(t)=A(t)+\widetilde{A}(t)$. Then, Theorem 1 holds under the same Assumptions A1-A5 with the replacement

$$
\beta_{1} \longrightarrow \beta_{1}^{\prime}=\beta_{1}+\sup _{t \geq t_{0}}\|B(t)-A(t)\|=\beta_{1}+\sup _{t \geq t_{0}} \sup _{\|z\|=1} \frac{\|(B(t)-A(t)) z\|}{\|z\|},
$$

where the vector-induced matrix norm $\|X\|=\sup _{\|z\|=1}(\|X z\| /\|z\|)$ is used.

Corollary 2. Consider the following controlled differential system:

$$
\dot{x}(t)=F(t, x)+u(t, x),
$$

subject to initial conditions $x\left(t_{0}\right)=x_{0}$, where $u:\left[t_{0}, \infty\right) \longrightarrow \mathbf{R}^{m}$ is a linear feedback control defined by $u(t, x)=K(t) x(t)$, where $K:\left[t_{0}, \infty\right) \times \mathbf{R}^{n} \longrightarrow \mathbf{R}^{m}$ is a bounded piecewise continuous matrix control gain and $F(t, x)$ is defined by (18) such that $\dot{z}(t)=(B(t)+K(t)) z(t)$ is exponentially stable with stability abscissa $-\alpha<0$. Then, Theorem 1 holds with $A(t)=B(t)+K(t)$.
Corollary 3. Assume that in Corollary 2, $u:\left[t_{0}, \infty\right) \times \mathbf{R}^{n} \longrightarrow L_{\infty}^{n}\left[t_{0}, \infty\right)$, where $L_{\infty}^{n}\left[t_{0}, \infty\right)$ is the set of essentially bounded real $n$-vector functions in $t$ on $\left[t_{0}, \infty\right)$ for all $x \in \mathbf{R}^{n}$ and let $u$ define $\bar{u}=\sup _{x \in \mathbf{R}^{n}} \int_{t_{0}}^{\infty}\|u(\tau, x)\| d \tau$. Then, Theorem 1 still holds by replacing (4) by

$$
\sum_{i=1}^{p} \beta_{i} r^{i-1}+\sum_{i=1}^{q} \gamma_{i} r^{i-1}+\frac{\bar{u}}{r} \leq \frac{\alpha}{K}
$$

and (5) by its corresponding strict inequality.

Proof. It follows directly by replacing (8) and (9) by

$$
\begin{gathered}
x(t)=\Psi\left(t, t_{0}\right) x_{0}+\int_{t_{0}}^{t} \Psi(t, \tau)\left(\sum_{i=1}^{p} f_{i}(\tau, x(\tau))+\sum_{i=1}^{q} \int_{t_{0}}^{t} g_{i}(\tau, x(\tau))+u(\tau, x(\tau))\right) \mathrm{d} \tau, \quad t \geq t_{0}, \\
\|x(t)\| \leq K e^{-\alpha\left(t-t_{0}\right)}\left(\left\|x_{0}\right\|-\frac{r}{\alpha}\left(\sum_{i=1}^{p} \beta_{i} r^{i-1}+\sum_{i=1}^{q} \gamma_{i} r^{i-1}\right)\right)+\frac{K r}{\alpha}\left(\sum_{i=1}^{p} \beta_{i} r^{i-1}+\sum_{i=1}^{q} \gamma_{i} r^{i-1}+\frac{\bar{u}}{r}\right) \leq r, \quad t \geq t_{0},
\end{gathered}
$$

and following similar arguments to those used to prove Theorem 1.

The following uniform-type result within a bounded ball of the solution of (1) and (2) follows from Theorem 1:
Corollary 4. Assume that A4 is modified by replacing (4) with

$$
\left(A 4^{\prime}\right) \max _{1 \leq i \leq p} \beta_{i}+\max _{1 \leq i \leq q} \gamma_{i} \leq \frac{\alpha}{\left(K M_{R}\right)}
$$


where

$$
\begin{gathered}
M_{R}=p M_{R p}+q M_{R q}, \\
M_{R p}=\max \left(1, R, \ldots, R^{p-1}\right), \\
M_{R q}=\max \left(1, R, \ldots, R^{q-1}\right) .
\end{gathered}
$$

Then, the following properties hold under Assumptions $A 1-A 3$ and $A 4$ : (i) If $\left\|x_{0}\right\| \leq(r / K)$ then $\sup _{t_{0} \leq t \leq \infty}\|x(t)\| \leq r$ for any given $r \in B_{R}=\left\{z \in \mathbf{R}_{+}\right.$: $\left.\|z\| \leq R\right\}$ so that differential system (1) and (2) is globally Lyapunov stable in $B_{R}$.

(ii) Assume that A5 is modified by replacing (5) with

$$
\left(\mathrm{A} 5^{\prime}\right) \max _{1 \leq i \leq p} \beta_{i}+\max _{1 \leq i \leq q} \gamma_{i}<\frac{\alpha}{\left(K M_{R}\right)} \text {. }
$$

Then, there is a unique solution of (1) and (2) for $t \geq t_{0}$ under Assumptions A1, A2, A3, and A5 satisfying

$$
\|x(t)\| \leq K e^{-\left(\alpha-K p M_{R p} \max _{1 \leq i \leq p} \beta_{i}\right)\left(t-t_{0}\right)}\left\|x_{0}\right\|+\frac{K q M_{R q} \max _{1 \leq i \leq q} \gamma_{i}}{\alpha-K p M_{R p} \max _{1 \leq i \leq p} \beta_{i}}\left(1-e^{-\left(\alpha-K p M_{R p}\right)\left(t-t_{0}\right)}\right) \leq r, \quad t \geq t_{0} .
$$

Outline of Proof. The proof follows directly from Theorem 1

by noting that (23) implies that

$$
\alpha \geq K M_{R}\left(\max _{1 \leq i \leq p} \beta_{i}+\max _{1 \leq i \leq q} \gamma_{i}\right) \geq K \max \left(p M_{R p} \max _{1 \leq i \leq p} \beta_{i}, q M_{R q} \max _{1 \leq i \leq q} \gamma_{i}\right)
$$

and (24) implies that

$$
\alpha>K M_{R}\left(\max _{1 \leq i \leq p} \beta_{i}+\max _{1 \leq i \leq q} \gamma_{i}\right) \geq K \max \left(p M_{R p} \max _{1 \leq i \leq p} \beta_{i}, q M_{R q} \max _{1 \leq i \leq q} \gamma_{i}\right)
$$

which makes (27) to be well-posed.

\section{Total Stability of Nonlinear Differential \\ Systems with Linear Constant Punctual \\ Delays and Forcing Terms Subject to \\ Combined Higher-Order Lipschitz Conditions}

Now, consider the following unforced linear time-invariant differential system subject to a finite set of $\vartheta$, in general, incommensurate constant point delays $0<h_{1}<h_{2}<\cdots<h_{\vartheta}=h$. These delays are commensurate in the particular case that $h_{i}=i h, i=1,2, \ldots, \vartheta$ (see, for instance, $[3,7]$ and some references therein). In the sequel, fix the initial time instant to $t_{0}=0$ for simplicity of the presentation of the subsequent results:

$$
\dot{x}(t)=A_{0} x(t)+\sum_{i=1}^{9} A_{i} x\left(t-h_{i}\right)
$$

with $A_{i} \in \mathbf{R}^{n \times n}, \quad i=0,1, \ldots, \vartheta$ which is subject to any bounded piecewise continuous function of initial conditions $\varphi:[t-h, 0] \longrightarrow \mathbf{R}^{n}$ with $\varphi(0)=x_{0}$ whose unique solution becomes to be

$$
\begin{aligned}
x(t) & =e^{A_{0} t}\left(x_{0}+\sum_{i=1}^{\vartheta} \int_{0}^{t} e^{-A_{0} \tau} A_{i} x\left(\tau-h_{i}\right) \mathrm{d} \tau\right) \\
& =e^{A_{0} t}\left(x_{0}+\sum_{i=1}^{\vartheta} \int_{0}^{h_{i}} e^{-A_{0} \tau} A_{i} \varphi\left(\tau-h_{i}\right) \mathrm{d} \tau+\sum_{i=1}^{\vartheta} \int_{h_{i}}^{t} e^{-A_{0} \tau} A_{i} x\left(\tau-h_{i}\right) \mathrm{d} \tau\right) \\
& =e^{A_{0} t}\left(x_{0}+\sum_{i=1}^{\vartheta} \int_{0}^{h_{i}} e^{-A_{0} \tau} A_{i} \varphi\left(\tau-h_{i}\right) \mathrm{d} \tau+e^{-A_{0} h_{i}} \sum_{i=1}^{\vartheta} \int_{0}^{t-h_{i}} e^{-A_{0} \tau} A_{i} x(\tau) \mathrm{d} \tau\right),
\end{aligned}
$$


where $\left\|e^{A_{0} t}\right\| \leq K e^{-\alpha t}$ for all $t \geq 0$ and some real constants $\alpha$ and $K \geq 1$ so that

$$
\begin{aligned}
\|x(t)\| & \leq K e^{-\alpha t}\left[\left\|x_{0}\right\|+\frac{1}{\alpha} \sum_{i=1}^{\vartheta}\left\|A_{i}\right\|\left(e^{\alpha h_{i}}-1\right) \sup _{-h \leq \tau \leq 0}\|\varphi(\tau)\|\right]+K e^{-\alpha t} \sum_{i=1}^{\vartheta} e^{\alpha h_{i}}\left\|A_{i}\right\| \int_{0}^{t-h_{i}} e^{\alpha \tau}\|x(\tau)\| \mathrm{d} \tau \\
& \leq K e^{-\alpha t}\left(1+\frac{1}{\alpha} \sum_{i=1}^{\vartheta}\left\|A_{i}\right\|\left(e^{\alpha h_{i}}-1\right)\right) \bar{\varphi}_{0}+\frac{K}{\alpha} \sum_{i=1}^{\vartheta}\left(1-e^{-\alpha\left(t-h_{i}\right)}\right)\left\|A_{i}\right\| \sup _{0 \leq \tau \leq t-h}\|x(\tau)\|,
\end{aligned}
$$

where $\bar{\varphi}_{0}=\sup _{-h \leq \tau \leq 0}\|\varphi(\tau)\|$.

Above differential system (30) can be rewritten equivalently as follows after introducing the zero delay $h_{0}=0$ and the matrix $A=\sum_{i=0}^{\vartheta} A_{i}$ :

$$
\dot{x}(t)=A x(t)+\sum_{i=1}^{\vartheta} A_{i}\left(x\left(t-h_{i}\right)-x(t)\right) .
$$

Note from (30) that the auxiliary differential system $\dot{x}(t)=A_{0} x(t)$ describes the time-delay system as $h_{i} \longrightarrow \infty \quad(i=1,2, \ldots, 9)$, which can be interpreted alternatively as the delay-free one when the contributions of delayed dynamics are zeroed described by $A_{i}=0$; $i=1,2, \ldots, \vartheta$. Also, note from (33) that the auxiliary differential system $\dot{x}(t)=A x(t)$ describes the time-delay system as $h_{i}=0 \quad(i=1,2, \ldots, \vartheta)$. It follows that the solution may be equivalently defined by

$$
x(t)=e^{A t}\left(x_{0}+\sum_{i=1}^{\vartheta} \int_{0}^{h_{i}} e^{-A \tau} A_{i}\left(\varphi\left(\tau-h_{i}\right)-\varphi(\tau)\right) \mathrm{d} \tau+\sum_{i=1}^{\vartheta} \int_{h_{i}}^{t} e^{-A \tau} A_{i}\left(x\left(\tau-h_{i}\right)-x(\tau)\right) \mathrm{d} \tau\right)
$$

where $\left\|e^{A t}\right\| \leq \bar{K} e^{-\bar{\alpha} t}$ for all $t \geq 0$ and some real constants $\bar{\alpha}$ and $\bar{K} \geq 1$ so that

$$
\begin{aligned}
\|x(t)\| & \leq K e^{-\bar{\alpha} t}\left[\left\|x_{0}\right\|+\frac{2}{\bar{\alpha}} \sum_{i=1}^{\vartheta}\left\|A_{i}\right\|\left(e^{\bar{\alpha} h_{i}}-1\right) \sup _{-h \leq \tau \leq 0}\|\varphi(\tau)\|\right]+2 \bar{K} e^{-\alpha t} \sum_{i=1}^{\vartheta} e^{\alpha h_{i}}\left\|A_{i}\right\| \int_{0}^{t-h_{i}}\|x(\tau)\| e^{\alpha \tau} \mathrm{d} \tau \\
& \leq K e^{-\bar{\alpha} t}\left(1+\frac{2}{\bar{\alpha}} \sum_{i=1}^{\vartheta}\left\|A_{i}\right\|\left(e^{\bar{\alpha} h_{i}}-1\right)\right) \bar{\varphi}_{0}+\frac{2 \bar{K}}{\bar{\alpha}} \sum_{i=1}^{\vartheta}\left(1-e^{-\alpha\left(t-h_{i}\right)}\right)\left\|A_{i}\right\| \sup _{0 \leq \tau \leq t-h}\|x(\tau)\|,
\end{aligned}
$$

where $\bar{\varphi}_{0}=\sup _{-h \leq \tau \leq 0}\|\varphi(\tau)\|$.

The following auxiliary result holds.

\section{Proposition 1. The following properties hold:}

(i) Let $A_{0}$ be a stability matrix such that $\left\|e^{A_{0} t}\right\| \leq K e^{-\alpha t}$ for all $t \geq 0$ and some real constants $\alpha>0$ and $K \geq 1$ (with $K$ being norm-dependent) and fix $\bar{\varphi}_{0}=\lambda r$. If

$$
0 \leq \lambda \leq \lambda_{M}=\frac{\alpha+K \sum_{i=1}^{\vartheta}\left\|A_{i}\right\|\left(e^{\alpha h_{i}}-1\right)}{\left(\alpha+\sum_{i=1}^{\vartheta}\left\|A_{i}\right\|\left(e^{\alpha h_{i}}-1\right)\right) K}
$$

then $\|x(t)\| \leq r ; \forall t \geq 0$, and thus, the system is globally Lyapunov stable.

(ii) Let $A=\sum_{i=0}^{\vartheta} A_{i}$ be a stability matrix such that $\left\|e^{A t}\right\| \leq \bar{K} e^{-\bar{\alpha} t}$ for all $t \geq 0$ and some real constants $\bar{\alpha}>0$ and $\bar{K} \geq 1$ (with $\bar{K}$ being norm-dependent) and fix $\bar{\varphi}_{0}=\lambda r$. If

$$
0 \leq \bar{\lambda} \leq \bar{\lambda}_{M}=\frac{\bar{\alpha}+2 \bar{K} \sum_{i=1}^{\vartheta}\left\|A_{i}\right\|\left(e^{\bar{\alpha} h_{i}}-1\right)}{\left(\bar{\alpha}+2 \sum_{i=1}^{\vartheta}\left\|A_{i}\right\|\left(e^{\bar{\alpha} h_{i}}-1\right)\right) \bar{K}}
$$

then $\|x(t)\| \leq r ; \forall t \geq 0$, and this the system is globally Lyapunov stable.

Proof. Note that $\lambda \leq 1$ so that $\sup _{-h \leq \tau \leq 0}\|x(\tau)\|=$ sup $_{-h \leq \tau \leq 0}\|\varphi(\tau)\| \leq r$ and there exist some $t_{0}>0$ such that $\sup _{-h \leq \tau \leq t_{0}}\|x(\tau)\| \leq r$ by continuity of the solution. Now, proceed by complete induction by using (32). Assume that there is a first time instant $t_{1}(>0) \in[t-(i+1) h, t-i h)$ for some $i(\in \mathbf{Z}) \in\left[0, \max \operatorname{int}\left(z: z \leq\left(t_{1} / h\right)-1\right)\right]$ such that $\left\|x\left(t_{1}\right)\right\|>r$. However, this gives a contradiction since then 
$\sup _{-h \leq \tau<t_{1}}\|x(\tau-h)\|>r$ and then there is some $t_{2} \in\left(0, t_{1}-h\right)$ which is the first time instant such that $\left\|x\left(t_{2}\right)\right\|>r$. As a result, $\bar{\varphi}_{0} \leq\left(\alpha+K \sum_{i=1}^{\vartheta}\left\|A_{i}\right\|\left(e^{\alpha h_{i}}-1\right)\right) /\left(\alpha K+K \sum_{i=1}^{\vartheta}\left\|A_{i}\right\|\left(e^{\alpha h_{i}}-1\right)\right) r$ implies that $\sup _{t \geq-h}\|x(t)\| \leq r$ which proves Property (i). The proof of Property (ii) is similar to that of Property (i) by using (35) instead of (32).

Remark 1. Note that $\lambda_{M}$ and $\bar{\lambda}_{M}$ are strictly decreasing functions of any delay on the positive real axis. This is seen easily by considering only one delay $h=h_{1}$ and define $v(h)=$ $\left\|A_{i}\right\|\left(e^{\alpha h_{i}}-1\right)$ leading to $\lambda_{M}=\lambda_{M}(v)=\left(\alpha K^{-1}+v\right) /(\alpha+v)$. Assume that $h^{\prime}>h$, so that $v\left(h^{\prime}\right)>v(h)$ then $\lambda_{M}\left(v^{\prime}\right)<\lambda_{M}(v)$ since, equivalently, $0<\left(K^{-1}+1\right) \alpha\left(v^{\prime}(h)-v(h)\right)$. A similar conclusion follows for $\bar{\lambda}_{M}$. This concern is that we can expect from intuition; that is, the increase in the delay sizes translates into a more strict sufficiently type constraint on the "smallness" constraint on the initial conditions to guarantee that the solution is kept under a certain prescribed closed ball through time.

Two sufficiently type conditions of global asymptotic Lyapunov stability at exponential rate of (30) are obtained in the next result.
Proposition 2. The following properties hold:

(i) Assume that $A_{0}$ is a stability matrix such that $\left\|e^{A_{0} t}\right\| \leq K e^{-\alpha t}$ for all $t \geq 0$ and some real constants $\alpha>0$ and $K \geq 1$. Then, differential system (30) is exponentially stable (i.e., for any given bounded piecewise continuous function of initial conditions) if $\alpha>K \sum_{i=1}^{9} e^{\alpha h_{i}}\left\|A_{i}\right\|$ which is guaranteed for any delays $0<h_{i}<h_{i+1} ; \quad i=1,2, \ldots, \vartheta-1$ satisfying $h_{\vartheta}<(1 /$ $\alpha) \ln \left(\alpha / K \vartheta \sum_{i=1}^{\vartheta}\left\|A_{i}\right\|\right)$.

(ii) Assume that $A=\sum_{i=0}^{\vartheta} A_{i}$ is a stability matrix such that $\left\|e^{A t}\right\| \leq \bar{K} e^{-\bar{\alpha} t}$ for all $t \geq 0$ and some real constants $\bar{\alpha}>0$ and $K \geq 1$ so that the delay-free version of (30) is exponentially stable. Then, differential system (30) is exponentially stable if $\bar{\alpha}>2 \bar{K} \sum_{i=1}^{\vartheta} e^{\bar{\alpha} h_{i}}\left\|A_{i}\right\|$ which is guaranteed for any delays $0<h_{i}<h_{i+1} ; i=1,2, \ldots$, $\vartheta-1$ satisfying $h_{\vartheta}<(1 / \bar{\alpha}) \ln \left(\bar{\alpha} / 2 \bar{K} \vartheta \sum_{i=1}^{\vartheta}\left\|A_{i}\right\|\right)$.

Proof. From the first inequality in (32), it follows that

$$
v(t)=\|x(t)\| e^{\alpha t} \leq K\left[\left\|x_{0}\right\|+\frac{1}{\alpha} \sum_{i=1}^{\vartheta}\left\|A_{i}\right\|\left(e^{\alpha h_{i}}-1\right) \sup _{-h \leq \tau \leq 0}\|\varphi(\tau)\|\right]+K \sum_{i=1}^{\vartheta} e^{\alpha h_{i}}\left\|A_{i}\right\| \int_{0}^{t} v(\tau) \mathrm{d} \tau, \quad \forall t \geq 0
$$

so that, from Gronwall Lemma [1-3],

$$
v(t) \leq K\left[\left\|x_{0}\right\|+\frac{1}{\alpha} \sum_{i=1}^{\vartheta}\left\|A_{i}\right\|\left(e^{\alpha h_{i}}-1\right) \sup _{-h \leq \tau \leq 0}\|\varphi(\tau)\|\right] e^{K \sum_{i=1}^{\vartheta} e^{\alpha h_{i}}\left\|A_{i}\right\| t}, \quad \forall t \geq 0,
$$

and then

$$
\|x(t)\| \leq K\left[1+\frac{1}{\alpha} \sum_{i=1}^{\vartheta}\left\|A_{i}\right\|\left(e^{\alpha h_{i}}-1\right)\right] \sup _{-h \leq \tau \leq 0}\|\varphi(\tau)\| e^{-\left(\alpha-K \sum_{i=1}^{\vartheta} e^{\alpha h_{i}}\left\|A_{i}\right\|\right) t}, \quad \forall t \geq 0
$$

On the other hand, one obtains from the first inequality in (35) and Gronwall Lemma that

$$
\|x(t)\| \leq \bar{K}\left[1+\frac{2}{\bar{\alpha}} \sum_{i=1}^{\vartheta}\left\|A_{i}\right\|\left(e^{\bar{\alpha} h_{i}}-1\right)\right] \sup _{-h \leq \tau \leq 0}\|\varphi(\tau)\| e^{-\left(\bar{\alpha}-2 \bar{K} \sum_{i=1}^{\vartheta} e^{\bar{\alpha} h_{i}}\left\|A_{i}\right\|\right) t}, \quad \forall t \geq 0 .
$$

Property (i) follows from (40) if $A_{0}$ is stability matrix while Property (ii) follows from (41) if $A$ is a stability matrix.

The extensions of Propositions 1 and 2 to the time-varying linear case are direct as follows in the subsequent result:
Proposition 3. The following properties hold if differential system (30) is time-varying:

(i) Assume that 
(A6) $A_{i}:[0, \infty) \longrightarrow \mathbf{R}^{n \times n}, \quad(i=0.1, \ldots, 9)$ have piecewise continuous and bounded entries.

(A7) the unforced system $\dot{z}(t)=A_{0}(t) z(t)$ is exponentially stable, that is, its fundamental matrix of $\Psi_{0}(t, 0)$, such that $\dot{\Psi}_{0}(t, 0)=A(t) \Psi_{0}(t, 0)$ with $\Psi_{0}(0,0)=I_{n}$, satisfies $\left\|\Psi_{0}(t, 0)\right\| \leq K e^{-\alpha t}$ for all $t \geq 0$ with real constants $K \geq 1$ and $\alpha>0$.

Thus, if $\quad \bar{\varphi}_{0}=\sup _{-h \leq \tau \leq 0}\|\varphi(\tau)\| \leq\left(\alpha+K \sum_{i=1}^{\mathcal{Q}}\right.$ $\left.\sup _{t>0}\left\|A_{i}(t)\right\|\left(e^{\alpha h_{i}}-1\right)\right) /\left(\left(\alpha+\sum_{i=1}^{\vartheta} \sup _{t \geq 0}\left\|A_{i}(t)\right\|\right.\right.$ $\left.\left.\left(e^{\alpha h_{i}}-1\right)\right) K\right) r$ then $\|x(t)\| \leq r ; \forall t>0$. Also, if $\alpha>K \sum_{i=1}^{\vartheta} e^{\alpha h_{i}}\left\|A_{i}\right\|$, then differential system (30) is globally asymptotically stable, with the solution trajectory being constrained to the ball $\|x(t)\| \leq r$; $\forall t>0$, and $x(t) \longrightarrow 0$ at exponential rate $-(\alpha-$ $\left.K \sum_{i=1}^{\vartheta} e^{\alpha h_{i}}\left\|A_{i}\right\|\right)$ as $t \longrightarrow \infty$.

(ii) Assume that Assumption A6 holds and, furthermore,

(A8) the unforced system $\dot{z}(t)=A(t) z(t)$ is exponentially stable, that is, its fundamental matrix of $\Psi(t, 0)$, such that $\dot{\Psi}(t, 0)=A(t) \Psi(t, 0)$ with $\Psi(0,0)=I_{n}$, satisfies $\|\Psi(t, 0)\| \leq \bar{K} e^{-\bar{\alpha} t}$ for all $t \geq 0$ with real constants $\bar{K} \geq 1$ and $\bar{\alpha}>0$.
Thus, if $\bar{\varphi}_{0}=\sup _{-h \leq \tau \leq 0}\|\varphi(\tau)\| \leq\left(\bar{\alpha}+2 \bar{K} \sum_{i=1}^{\vartheta} \sup _{t \geq 0} \| A_{i}\right.$ $\left.(t) \|\left(e^{\bar{\alpha} h_{i}}-1\right)\right) /\left(\left(\bar{\alpha}+2 \sum_{i=1}^{\vartheta} \sup _{t \geq 0}\left\|A_{i}(t)\right\|\left(e^{\bar{\alpha} h_{i}}-1\right)\right) \bar{K}\right) r$,

then $\|x(t)\| \leq r ; \forall t>0$. Also, if $\alpha>\bar{K} \sum_{i=1}^{\vartheta} e^{\bar{\alpha} h_{i}}\left\|A_{i}\right\|$, then differential system (30) is exponentially stable, with the solution trajectory being constrained to the ball $\|x(t)\| \leq r ; \forall t>0$, so that $x(t) \longrightarrow 0$ at exponential rate $-\left(\bar{\alpha}-\bar{K} \sum_{i=1}^{\vartheta} e^{\alpha h_{i}}\left\|A_{i}\right\|\right)$ as $t \longrightarrow \infty$.

The following main total stability result gives sufficiencytype global stability conditions of the delayed system in the presence of nonlinearities which generalize those considered in Theorem 1.

Theorem 2. Consider the following differential system:

$$
\dot{x}(t)=F\left(t, x(t), x\left(t-h_{1}\right), x\left(t-h_{2}\right), \ldots, x\left(t-h_{\vartheta}\right)\right),
$$

with $A_{i} \in \mathbf{R}^{n \times n} \quad(i=0,1, \ldots, 9)$ which is subject to any bounded piecewise continuous function of initial conditions $\varphi:[t-h, 0] \longrightarrow \mathbf{R}^{n} \quad$ with $\quad \varphi(0)=x_{0} \quad$ where $F:[0, \infty) \times \mathbf{R}^{n} \longrightarrow \mathbf{R}^{n}$ is given by

$$
\begin{aligned}
F(t, x)= & A_{0}(t) x(t)+\sum_{i=1}^{\vartheta} A_{i}(t) x\left(t-h_{i}\right) \\
& +\sum_{i=1}^{p} f_{i}\left(t, x(t), x\left(t-h_{1}\right), \ldots, x\left(t-h_{\vartheta}\right)\right)+\sum_{i=1}^{q} g_{i}\left(t, x(t), x\left(t-h_{1}\right), \ldots, x\left(t-h_{\vartheta}\right)\right),
\end{aligned}
$$

where $A_{i}:\left[t_{0}, \infty\right) \longrightarrow \mathbf{R}^{n \times n} \quad(i=1,2, \ldots, 9)$ have piecewise continuous entries and $f_{i}, g_{j}:\left[t_{0}, \infty\right) \times \mathbf{R}^{(9+1) n} \longrightarrow \mathbf{R}^{n}$; $i=1,2, \ldots, p ; j=1,2, \ldots, q$ are locally integrable functions of $t$ on $[0, \infty]$ in the closed ball $B_{r}=\left\{x \in \mathbf{R}^{n}:\|x\| \leq r\right\}$. Suppose that Assumption A6 and that furthermore the following assumptions hold for some given $r>0$ :

$$
\begin{aligned}
& \text { (A9) } f_{i}(t, 0, \ldots, 0)=0 \\
& \left\|f_{i}\left(t, x_{1}, x_{2}, \ldots, x_{\vartheta+1}\right)-f_{i}\left(t, y_{1}, y_{2}, \ldots, y_{\vartheta+1}\right)\right\| \leq \sum_{j=0}^{\vartheta} \beta_{i j k}\left\|x_{j}-y_{k}\right\|^{i}, \quad i=1,2, \ldots, p \text {, for all } x_{j}, y_{k} \in B_{r}, \\
& (\mathrm{~A} 10)\left\|g_{i}\left(t, x_{1}, x_{2}, \ldots, x_{\vartheta+1}\right)\right\| \leq \sum_{j=0}^{\vartheta} \sum_{k=0}^{\vartheta} \gamma_{i j k} r^{i+1}, \\
& \left\|g_{i}\left(t, x_{1}, x_{2}, \ldots, x_{\vartheta+1}\right)-g_{i}\left(t, y_{1}, y_{2}, \ldots, y_{\vartheta+1}\right)\right\| \leq \sum_{j=0}^{\vartheta} \sum_{k=0}^{\vartheta} \gamma_{i j k}\left\|x_{j}-y_{k}\right\|^{i}, \quad i=1,2, \ldots, q, \text { for all } x_{j}, y_{k} \in B_{r},
\end{aligned}
$$

(A11) $\alpha>\max \left(\alpha_{1}, \alpha_{2}\right)$

which is guaranteed if $\alpha>\alpha_{1}+\alpha_{2}$, where 


$$
\begin{gathered}
\alpha_{1}>K \sum_{i=1}^{9} \sup _{t \geq 0}\left\|A_{i}(t)\right\|\left(e^{\alpha h_{i}}-1\right), \\
\alpha_{2}>K\left(\sum_{i=1}^{p} \sum_{j=0}^{9} \sum_{k=0}^{9} \beta_{i j k} r^{i-1}+\sum_{i=1}^{q} \sum_{j=0}^{9} \sum_{k=0}^{9} \gamma_{i j k} r^{i-1}\right), \\
\bar{\varphi}_{0}=\sup _{-h \leq \tau \leq 0}\|\varphi(\tau)\| \leq \frac{\alpha-K\left(\sum_{i=1}^{p} \sum_{j=0}^{9} \sum_{k=0}^{9} \beta_{i j k} r^{i-1}+\sum_{i=1}^{q} \sum_{j=0}^{\vartheta} \sum_{k=0}^{\vartheta} \gamma_{i j k} r^{i-1}\right)}{\left(\alpha+\sum_{i=1}^{\vartheta} \sup _{t \geq 0}\left\|A_{i}(t)\right\|\left(e^{\alpha h_{i}}-1\right)\right) K} r .
\end{gathered}
$$

Then, the following properties hold:

(i) There is a unique globally Lyapunov stable solution of (42) and (43) satisfying

$$
\begin{aligned}
\|x(t)\| \leq & K\left[1+\frac{1}{\alpha} \sum_{i=1}^{9}\left\|A_{i}\right\|\left(e^{\alpha h_{i}}-1\right)\right] \sup _{h \leq \tau \leq 0}\|\varphi(\tau)\| e^{-\left(\alpha-K\left(\sum_{i=1}^{9} e^{\alpha h_{i}} \sup _{\tau \geq 0}\left\|A_{i}(\tau)\right\|+\sum_{i=1}^{p} \sum_{j=0}^{9} \sum_{k=0}^{9} \beta_{i j k} r^{i-1}\right)\right) t} \\
& +\frac{K \sum_{i=1}^{q} \sum_{j=0}^{9} \sum_{k=0}^{9} \gamma_{i j k} r^{i}}{\alpha-K \sum_{i=1}^{p} \sum_{j=0}^{9} \sum_{k=0}^{9} \beta_{i j k} r^{i-1}}\left(1-e^{-\left(\alpha-K\left(\sum_{i=1}^{9} e^{\alpha h_{i}} \sup _{\tau \geq 0}\left\|A_{i}(\tau)\right\|+\sum_{i=1}^{p} \sum_{j=0}^{9} \sum_{k=0}^{9} \beta_{i j k} r^{i-1}\right)\right) t}\right) \leq r, \quad \forall t \geq 0 .
\end{aligned}
$$

(ii) Assume that $\alpha, \alpha_{1}$, and $\alpha_{2}$ and $\bar{\varphi}_{0}$ in (45)-(47) are replaced with similar equations under the replacements $\quad \alpha_{1} \longrightarrow \bar{\alpha}_{1}=2 \bar{K} \sum_{i=1}^{\vartheta} \sup _{t \geq 0}\left\|A_{i}(t)\right\|\left(e^{\alpha h_{i}}-1\right)$, $\alpha_{2} \longrightarrow \bar{\alpha}_{2}$ (obtained by replacing $K \longrightarrow \bar{K}$ ), Assumption A1 being modified with the replacement $\alpha \longrightarrow \bar{\alpha}>\max \left(\bar{\alpha}_{1}, \bar{\alpha}_{2}\right)$ and (47) being modified with the replacements $\alpha \longrightarrow \bar{\alpha}, \quad K \longrightarrow \bar{K}, \quad$ and $\sup _{t \geq 0}\left\|A_{i}(t)\right\| \longrightarrow 2 \sup _{t \geq 0}\left\|A_{i}(t)\right\|$ in the denominator of (47) and to define. Then, there is a unique globally Lyapunov stable solution of (42) and (43) satisfying

$$
\begin{aligned}
\|x(t)\| \leq & \bar{K}\left[1+\frac{2}{\bar{\alpha}} \sum_{i=1}^{9}\left\|A_{i}\right\|\left(e^{\bar{\alpha} h_{i}}-1\right)\right] \sup _{h \leq \tau \leq 0}\|\varphi(\tau)\| e^{-\left(\bar{\alpha}-2 \bar{K}\left(\sum_{i=1}^{\vartheta} e^{\bar{\alpha} h_{i}} \sup _{\tau \geq 0}\left\|A_{i}(\tau)\right\|+\sum_{i=1}^{p} \sum_{j=0}^{\vartheta} \sum_{k=0}^{\vartheta} \beta_{i j k} r^{i-1}\right)\right) t} \\
& +\frac{\bar{K} \sum_{i=1}^{q} \sum_{j=0}^{\vartheta} \sum_{k=0}^{\vartheta} \gamma_{i j k} r^{i}}{\bar{\alpha}-\bar{K} \sum_{i=1}^{p} \sum_{j=0}^{9} \sum_{k=0}^{9} \beta_{i j k} r^{i-1}}\left(1-e^{-\left(\bar{\alpha}-2 \bar{K}\left(\sum_{i=1}^{9} e^{\bar{\alpha} h_{i}} \sup _{\tau \geq 0}\left\|A_{i}(\tau)\right\|+\sum_{i=1}^{p} \sum_{j=0}^{9} \sum_{k=0}^{9} \beta_{i j k} r^{i-1}\right)\right) t}\right) \leq r, \quad \forall t \geq 0 .
\end{aligned}
$$

Proof. It follows directly by combining Propositions 2 and 3 for differential system (42) and (43) with a similar technique of proof of Theorem 1 .

Remarks 2. (1) It is convenient now to summarize at a first glance which are the basic ideas behind the above results concerning the studied time-delay system.
(2) Note that Proposition 2 gives two results of global asymptotic Lyapunov stability in the large (i.e., for any bounced piecewise continuous function of initial conditions). In this way, the solution trajectory converges asymptotically to the zero equilibrium point at exponential rate. The result is independent of the delay size type if the delays are small enough provided that either the auxiliary system with delay- 
free dynamics of that with zero delay is exponentially stable.

(3) Note also that Proposition 1 gives two results of global Lyapunov stability independent of the delay sizes for a certain range of values of the delays with the trajectory solution being constrained within a certain closed ball around the origin for $t>0$ sufficiently small initial conditions provided that one of the above auxiliary unforced systems is exponentially stable.

Proposition 3 states results on global stability and global asymptotic stability with the trajectory being constrained to as ball around the origin if one of the two mentioned auxiliary systems is exponentially stable.

(4) Theorem 2 gives two alternative results of solution uniqueness and global stability within a ball around the origin, based on either the stability of the matrix $A_{0}$, corresponding to the system without delayed dynamics, or on the stability of the delay-free system of matrix dynamics $A$, in the case that systems (42) and (43) become forced under forcing functions with eventual delays which satisfy general nonlinear constraints based on extending those invoked in Theorem 1 for the delay-free system. Both results are supported by the constraints that the stability of the matrices $A_{0}$ and $A$ guarantee, respectively, the exponential stability of their respective unforced systems independent of the delay sizes within a range of values with a maximum bound.

In particular, Assumption A11 has a direct interpretation as follows: firstly, $\alpha>\alpha_{1}$ guarantees that the exponential stability of the unforced differential system $\dot{z}(t)=A_{0} z(t)$, which describes the case of absence of delayed dynamics, under any finite bounded conditions $z(0)=z_{0}$, guarantees that of system (30) for any bounded piecewise continuous function of initial conditions involving point delays for a certain maximum allowable size of the maximum delay. Secondly, if furthermore, in (46), $\alpha_{2}$ is small enough to satisfy $\alpha_{2}<\alpha-\alpha_{1}$, for given forcing functions $f_{(\cdot)}, g_{(\cdot)}$ subject to Assumptions A9 and A10, i.e., such that Assumption A11, is also satisfied; then, the forcing system remains globally Lyapunov stable within the closed ball of radius $r$ centred at the origin and satisfies (48). The alternative constraints invoked to get (49) are interpreted in a similar way based on the assumption of exponential stability under any finite initial conditions of the unforced delay-free differential system $\dot{z}(t)=A z(t)$, which describes the zerodelay case, that is, the case when $h_{i}=0$ for $i=1,2, \ldots, \vartheta$.

For the following discussion, recall that $(F, G)$ is a controllable pair with $F \in \mathbf{R}^{n \times n}$ and $G \in \mathbf{R}^{m \times n}$ if and only if $\operatorname{rank}\left(G, F G, \ldots, F^{n-1} G\right)=n$, equivalently, if and only if $\operatorname{rank}\left[s I_{n}-F, G\right]=n$ for any $s \in \mathbf{C}$ which is not an eigenvalue of $F$ (the Popov-Belevitch-Hautus controllability test). If $(F, G)$ is controllable, then the spectrum of $F+G K$ can be prefixed arbitrarily through the choice of $K \in \mathbf{R}^{m \times n}$ [4-6].

On the other hand, $(F, G)$ is a stabilizable pair if and only if $\operatorname{rank}\left[s I_{n}-F, G\right]=n$ for any $s \in \mathbf{C}$ with $\operatorname{Res} \geq 0$ which is not an eigenvalue of $F$ (the Popov-Belevitch-Hautus stabilizability test). If $(F, G)$ is stabilizable, then the spectrum of $F+G K$ can be stabilized (but it cannot be either arbitrarily assigned or prefixed subject to a prefixed stability abscissa) through the choice of $K \in \mathbf{R}^{m \times n}$ so that, for some $K \in \mathbf{R}^{m \times n}$, all the eigenvalues of $F+G K$ can be allocated in the open complex left-hand-side plane $\operatorname{Res}<0,[4,5]$.

It turns out that if $(F, G)$ is controllable, then it is stabilizable, but the converse is not true.

The subsequent results rely on the stabilization of (42) and (43) though a linear feedback control of the form $u(t)=K_{0} x(t)$.

Corollary 5. Assume that $B_{0} \in \mathbf{R}^{n \times m}$, with $m \leq n$, are constant matrices and that the pair $\left(A_{0}, B_{0}\right)$ is controllable and let differential system (42) be defined by

$$
\begin{aligned}
F(t, x)= & A_{0} x(t)+B_{0} K_{0} x(t)+\sum_{i=1}^{\vartheta} A_{i}(t) x\left(t-h_{i}\right) \\
& +\sum_{i=1}^{p} f_{i}\left(t, x(t), x\left(t-h_{1}\right), \ldots, x\left(t-h_{\vartheta}\right)\right)+\sum_{i=1}^{q} g_{i}\left(t, x(t), x\left(t-h_{1}\right), \ldots, x\left(t-h_{\vartheta}\right)\right),
\end{aligned}
$$

under the assumptions on the initial conditions and matrices of dynamics of Theorem 2 and Assumptions A9-A10 for any set of $\vartheta$ point delays $0<h_{1}<h_{2}<\cdots<h_{9} \leq h<+\infty$ with any associated matrices of dynamics $\sup _{\tau \geq 0} \max _{1 \leq i \leq 9}\left\|A_{i}(\tau)\right\| \leq a<+$ $\infty$ and any nonnegative real constants $\beta_{i j k} \leq \beta<+\infty$, $\gamma_{\ell j k} \leq \gamma<+\infty ; \quad i=1,2, \ldots, p ; \quad \ell=1,2, \ldots, q$; $j, k=1,2, \ldots, \vartheta$. Then, for any given quadruple $(h, a, \beta, \gamma)$ of nonnegative real constants, there exists a matrix $K_{0} \in \mathbf{R}^{m \times n}$ such that differential systems (42) and (43) are globally Lyapunov stable with its unforced $s$ version being exponentially stable.
Proof. Since $\left(A_{0}, B_{0}\right)$ is controllable, the spectrum (and then the stability abscissa) of any given matrix $\Lambda_{0} \in R^{n \times n}$ can be fixed by some existing $K_{0} \in \mathbf{R}^{m \times n}$ which satisfies the matrix equality $A_{0}+B_{0} K_{0}=\Lambda_{0}$. So, for any given triple $(a, \beta, \gamma)$, it is possible to fix a stability abscissa $\left(-\alpha_{\Lambda_{0}}\right)<0$ of some $\Lambda_{0}$ which satisfies Assumption A11, and then Theorem 2 (i) holds.

Under stabilizability conditions, rather than controllability conditions, of the pair $\left(A_{0}, B_{0}\right)$, a weaker result than Corollary 5 is now given since the stability abscissa of $A_{0}+$ $B_{0} K_{0}$ cannot be arbitrarily prefixed. 
Corollary 6. Assume the assumptions of Corollary 5 except for that the pair $\left(A_{0}, B_{0}\right)$ is stabilizable rather than controllable. Then, a quintuple $\left(h, a, \beta, \gamma, K_{0}\right)$ can be fixed such that differential systems (42) and (43) are globally Lyapunov stable with its unforced s version being exponentially stable.

The subsequent result relies on the stabilization of a linear time-delay system with a unique delay $h_{1}$ though a linear feedback control of the form $u(t)=K_{0} x(t)+K_{1} x(t-h)$. It is found that the exponential stability is achievable for large delay ranges if $\left(A_{0}, B\right)$ and $\left(A_{1}, B\right)$ are, respectively, stabilizable and controllable.

Corollary 7. Consider the following delayed unforced system:

$$
\dot{x}(t)=A x(t)+A_{1} x\left(t-h_{1}\right)+B u(t),
$$

with a control law $u(t)=K_{0} x(t)+K_{1} x(t-h)$ and assume that $B \in \mathbf{R}^{n \times m}$, with $m \leq n$ and some matrix feedback control gains $K_{0}, K_{1} \in \mathbf{R}^{m \times n}$. Assume that the pairs $\left(A_{0}, B\right)$ and $\left(A_{1}, B\right)$ are, respectively, stabilizable and controllable. Then, for any existing stabilizing matrix gain $K_{0}$ of the pair $\left(A_{0}, B\right)$, there exists a matrix gain $K_{1}$ such that the above system is exponentially stable for $h_{1} \in[0, \bar{h})$ and some $\bar{h}=\bar{h}\left(K_{1}\right)>0$ for each stabilizing control gain $K_{0}$ of $\left(A_{0}, B\right)$ and which depends on the control gain $K_{1}$. The stabilizing delay maximum bound $\bar{h}$ can be made arbitrarily large depending on the choice of such a control gain $K_{1}$.

Proof. Since $\left(A_{0}, B\right)$ is stabilizable, there is $K_{0}$ such that $\left(A_{0}+B_{0} K_{0}\right)$ is a stability matrix of some nonprefixed abscissa $-\alpha_{0}<0$. Now, from Proposition 2 , the above system is exponentially stable if $h_{1}<\left(1 / \alpha_{0}\right) \ln \left(\alpha_{0} / K\left\|A_{1}+B_{1} K_{1}\right\|\right)$ with $K(\geq 1)$ being norm-dependent. However, since $\left(A_{1}, B\right)$ is controllable, the whole spectrum of $A_{1}+B_{1} K$ is freely assignable by the choice of $K_{1}$; then, the spectral radius $\rho\left(A_{1}+B_{1} K_{1}\right)$ of $A_{1}+B_{1} K_{1}$, which is arbitrarily close to some matrix norm, can be chosen as small as possible so that the exponential stability holds for arbitrarily large delay according to the following formula:

$$
0 \leq h_{1}<\bar{h}=\frac{1}{\alpha_{0}} \ln \frac{\alpha_{0}}{K \rho\left(A_{1}+B_{1} K_{1}\right)+\varepsilon}=\frac{1}{\alpha_{0}} \ln \frac{\alpha_{0}}{K\left\|A_{1}+B_{1} K_{1}\right\|} .
$$

Corollary 8. Assume that $A_{0}:[0, \infty) \longrightarrow \mathbf{R}^{n \times n}$ is bounded piecewise continuous with $A_{0}(t)=A_{0 *}+\widetilde{A}_{0}(t)$ and that all the remaining assumptions of Corollary 5 hold with the modifications that $\left(A_{0 *}, B_{0}\right)$ is controllable and $\sup _{t \geq 0}\left(\max _{1 \leq i \leq 9}\left\|A_{i}(t)\right\|,\left\|\widetilde{A}_{0}(t)\right\|\right) \leq a<+\infty \quad$ and $\sup _{t \geq 0}\left(\max _{1 \leq i \leq 9}\left\|A_{i}(t)\right\|\right) \leq a<+\infty$. Then, for any given quadruple $(h, a, \beta, \gamma)$ of nonnegative real constants, there exists a matrix $K_{0} \in \mathbf{R}^{m \times n}$ such that differential systems (42) and (43) are globally Lyapunov stable with its unforced version being exponentially stable.

Proof. It is identical to that of Corollary 5 by adding a new (zero) delay $h_{0}=0$ with matrix of dynamics $\widetilde{A}_{0}(t)$ and replacing the lower-bound of $\alpha_{1}$ in (45) with the corresponding summation from $i=0$ to $i=\vartheta$, i.e., by incorporating the extra norm associated with $\sup _{t \geq 0}\left\|\widetilde{A}_{0}(t)\right\|$.

Remark 3. It turns out that a similar result to Corollary 8 can be directly formulated by considering that $\left(A_{0 *}, B_{0}\right)$ is stabilizable rather than controllable.

Remark 4. Extensions of Corollaries 5 to 7 and the direct result got as indicated in Remark 3 are direct from Theorem 2 (ii) for the cases of either controllability or stabilizability of the pair $(A, B)$ for a given $B \in \mathbf{R}^{m \times n}$ and $A=\sum_{i=0}^{\vartheta} A_{i} \in \mathbf{R}^{n \times n}$ describing the delay-free auxiliary system. If $A(t)$ is timevarying then, the problem can be stated by extending Theorem 2 (ii) with $A(t)=A_{*}+\widetilde{A}(t),\left(A_{*}, B\right)$ being controllable/stabilizable and $\widetilde{A}(t)$ being a disturbance matrix of zero delay (see Corollary 7 ).

It can be of interest to give some ideas about how the above results can be applied when switching through time between alternative active configurations of a time-delay system are performed. For this purpose, consider a nonempty set of $\Omega$ distinct configurations $S$ of (42) and (43) such that $S_{j}=\left(A_{j i}, f_{j k}, g_{j \ell}: i \in \bar{\vartheta} \cup\{0\}, k \in \bar{p}, q \in \bar{\ell}\right)$, with $\bar{n}=\{1,2, \ldots, n\}$, which admit switching between them through time given by a switching law $\sigma:[0, \infty) \longrightarrow \bar{\Omega}$ which is a piecewise continuous function. $T=\left\{t_{i}\right\}_{i=0}^{\chi}$, with $t_{0}=0$, is either a set or an infinite sequence (i.e., $\chi \leq \infty$ ) of switching instants between configurations; that is, $\sigma\left(t_{i}\right)=$ $\sigma\left(t_{i}^{+}\right) \neq \sigma\left(t_{i}^{-}\right)$and $\sigma(t)=\sigma\left(t_{i}\right)=j(\in \bar{\Omega}) ; \forall t \in\left[t_{i}, t_{i+1}\right)$, and it is said that $j$ is the active configuration on $\left[t_{i}, t_{i+1}\right)$. Assume that $S=S_{s} \cup S_{\bar{s}}$ such that

the configurations of $S_{s}$ satisfy Theorem 2 so that each corresponding unforced version of (42) and (43) is exponentially stable, so that it has a negative stability abscissa

the configurations of $S_{\bar{s}}$ are $S_{\bar{s}}=S_{c s} \cup S_{u s}$, where $S_{c s}$ have critically stable unforced versions so that each corresponding unforced version of (42) and (43) has a zero stability abscissa while the configurations of $S_{u s}$ are unstable so that each corresponding unforced version of (42) and (43) has a positive stability abscissa

Theorem 3. Assume that $S=S_{s} \cup S_{\bar{s}}$ with $S_{s} \neq \varnothing$ and that there is at least one $S^{*}=S_{i *} \in S_{s}$ with a pair $\left(K^{*},-\alpha^{*}\right)$ with $\alpha^{*}>0$ satisfying $\left(\alpha^{*} / K^{*}\right)>R$ for some given finite real constant $R>0$. Then, for any given admissible function of initial conditions satisfying $\bar{\varphi}_{0} \leq R$, there always exists switching laws $\sigma:[0, \infty) \longrightarrow \bar{\Omega}$ with a finite number of switches and switching time instants $\left\{t_{0}, t_{1}, \ldots, t_{k}\right\}$ such that $\sigma\left(t_{k}\right)=i^{*}$ and $\|x(t)\| \leq r_{i *} ; \forall t \geq t_{k}$.

Remark 5. Note that, in particular, $t_{1}=t_{i *}$ is such a switching law of only one or two switching time instants, the initial one at $t_{0}=0$, which can be at a distinct active configuration of $S_{i *}$ if $S \neq S_{i *}$ or directly at $S_{i *}$, necessarily if $S=S_{i *}$, and the second one at $t_{1}=t_{i *}$ which is only feasible if $S \neq S_{i *}$. 


\section{Data Availability}

No data were used to support this study.

\section{Conflicts of Interest}

The author declares that he does not have any conflicts of interest.

\section{Acknowledgments}

The author is grateful to the Spanish Government for grant RTI2018-094336-B-I00 (MCIU/AEI/FEDER, UE) and to the Basque Government for grant IT1207-19. He also thanks the Spanish Institute of Health Carlos III for its support through grant COV 20/01213.

\section{References}

[1] B. D. O. Anderson, R. R. Bitmead, C. R. Johnson Jr. et al., Stability of Adaptive Systems. Passivity and Averaging Analysis, The MIT Press, Cambridge, MA, USA, 1986.

[2] M. Delasen, "Use of Gronwall's Lemma for robust compensation of the time-varying linear systems via synthesis of augmented exciting signals," International Journal of Systems Science, vol. 21, no. 11, pp. 2317-2335, 1990.

[3] M. De la Sen and N. S. Luo, "A note on the stability of linear time-delay systems with impulsive inputs," IEEE Transactions on Circuits and Systems I- Regular Papers, vol. 50, no. 1, pp. 149-152, 2003.

[4] S. Barnett, Matrices in Control Theory with Applications to Linear Programming, Van Nostrand Reinhold Company, New York, NY, USA, 1971.

[5] S. Barnett, Polynomials and Linear Control Systems, Marcel Dekker, New York, NY, USA, 1983.

[6] S. Barnett, Introduction to Mathematical Control Theory, Oxford University Press, New York, NY, USA, 1990.

[7] C. Tunç and M. Gozen, "Stability and uniform boundedness in multidelay functional differential equations of third order," Abstract and Applied Analysis, vol. 2013, Article ID 248717, 7 pages, 2013.

[8] M. De la Sen, "On some sufficiency-type global stability results for time-varying dynamic systems with state-dependent parameterizations," International Journal of Differential Equations, vol. 2019, Article ID 5097974, 15 pages, 2019.

[9] C. Tunç, "On the stability and boundedness of solutions of nonlinear vector differential equations of third order," Nonlinear Analysis: Theory, Methods \& Applications, vol. 70, no. 6, pp. 2232-2236, 2009.

[10] Q. Wang, "On the construction of globally asymptotically stable Lyapunov functions for a type on nonlinear third-order system," Acta Mathematicae Applicatae Sinica, vol. 6, no. 3, pp. 309-323, 1983.

[11] T. Yoshizawa, "Asymptotic behaviour of solutions of a system of differential equations," Contributions to Differential Equations, vol. 1, pp. 371-387, 1963.

[12] D. Hinrichsen and A. J. Pritchard, "Robust exponential stability of time-varying linear systems," International Journal of Robust and Nonlinear Control, vol. 3, no. 1, pp. 63-83, 1993.

[13] T. A. Ademola, B. S. Ogundare, M. Ogundiran, and O. A. Adesina, "Stability, boundedness, and existence of periodic solutions to certain third-order delay differential equations with multiple deviating arguments," International
Journal of Differential Equations, vol. 2015, Article ID 213935 , 12 pages, 2015.

[14] B. S. Ogundare, J. A. Ayanjinmi, and O. A. Adesina, "Bounded and -solutions of certain third order non-linear differential equation with a square integrable forcing term," Kragujevac Journal of Mathematics, vol. 29, pp. 151-156, 2006.

[15] J. Andres and V. Vlcek, "Square integrable processes in nonlinear oscillators I," Acta UPO, vol. 92, pp. 91-104, 1991.

[16] J. Andres and V. Vlcek, "Square integrable processes in nonlinear oscillators II," Acta UPO, vol. 106, pp. 153-158, 1991.

[17] M. Conti, F. dell'Oro, and V. Pata, "Exponential decay of a first order linear Volterra equation," Mathematics in Engineering, vol. 2, no. 3, pp. 459-471, 2020.

[18] J. Xuelian, "Exponential stability analysis and control design for nonlinear systems with time-varying delay," AIMS Mathematics, vol. 6, no. 1, pp. 102-113, 2021.

[19] R. Dhayal, M. Malik, and S. Abbas, "Solvability and optimal controls of non-instantaneous impulsive stochastic neutral integro-differential equation driven by fractional Brownian motion," AIMS Mathematics, vol. 4, no. 3, pp. 663-683, 2019. 